\title{
InCLinAtion ANGLe EFFECT OF CARbon FibERS in Cementitious Composites
}

\author{
By Amnon Katz ${ }^{1}$ and Victor C. Li, ${ }^{2}$ Member, ASCE
}

\begin{abstract}
This paper presents an analytic model, which describes the bridging load and fiber stress developed when an inclined brittle fiber bridges a crack. The coupled effect of fiber bending and axial loading is explicitly accounted for. The model also considers matrix spalling and concentration of friction load at the point where the fiber bends over the matrix. With this model, it is possible to test the influence of fiber and matrix parameters on the bridging load and fiber stress, in order to achieve more efficient use of the fibers. Good comparison of the model predictions with experimental results of the inclined carbon-fiber test was achieved, both for the bridging load and the spall length. The model can serve as a useful tool to predict the efficiency of inclined fibers bridging a matrix crack.
\end{abstract}

\section{INTRODUCTION}

The inclination angle of a fiber bridging a matrix crack has been shown to have a significant effect on composite properties. Laws (1971) and Allen (1972) concluded that when a fiber is inclined toward the load direction, it bears only the component of load in its direction. Thus, the fiber efficiency is reduced for inclined fibers.

When a fiber bridges a matrix crack, as shown in Fig. 1, geometric restrictions lead to fiber bending. Friction concentration at the point at which the fiber exits the matrix increases the tensile stress in the fiber, leading to a more efficient use of the fiber. Li et al. (1990) showed this effect by using the pulley model to describe the angle effect on the pullout behavior of ductile fiber. In this model the fiber is simulated as a flexible rope pulled over a friction pulley. For stiff and brittle fiber, as the carbon fiber, this analogy may not be accurate as the fiber does not bend completely over the pulley due to its high stiffness. In addition, the fiber may break as a result of stress concentration caused by both the axial load of pullout and bending of fiber, as shown in Fig. 1.

Aveston et al. (1974) analyzed this problem for glass and carbon fibers. They concluded that there may be cases where the bending stress in the fiber is seven or 15 times larger than the axial load developed in the fiber. They also suggested that crumbling or yielding of the matrix below the fiber reduces the bending stress. However, they did not provide solutions to the extent of crumbling and its effect on the bending stress. Piggot (1974) considered the case of brittle fibers in a rigid perfectly plastic matrix. In this model the fiber is considered to be a flexible string with no bending stiffness, and the bending of the fiber bridging the crack due to geometric restrictions (as demonstrated in Fig. 1), was neglected.

Two models were developed by Katz (1992) and Leung and $\mathrm{Li}$ (1992) for modeling the behavior of stiff brittle fiber bridging over a crack. Katz (1992) adopted the model of Morton and Groves (1974), who treated the fiber as a cantilevered beam on an elastic foundation. Morton and Groves assumed a ductile fiber that yields plastically at the bending point. Leung and $\mathrm{Li}$ (1992) adopted a finite-element approach to take into account the three-dimensional nature of the physical

'Asst. Prof., Nat. Build. Res. Inst., Dept. of Civ. Engrg., TechnionIsrael Inst. of Technol., Haifa, 32000, Israel.

'Prof., Advanced Civ. Engrg. Mat. Res. Lab., Dept. of Civ. and Envir. Engrg., Univ. of Michigan, Ann Arbor, MI 48109-2125.

Note. Associate Editor: Robert Y. Liang. Discussion open until May 1, 1996. To extend the closing date one month, a written request must be filed with the ASCE Manager of Journals. The manuscript for this paper was submitted for review and possible publication on September 30, 1994. This paper is part of the Journal of Engineering Mechanics, Vol. 121, No. 12, December, 1995. CASCE, ISSN 0733-9399/95/0012$1340-1348 / \$ 2.00+\$ .25$ per page. Paper No. 9416 . problem, and also included the effect of fiber breakage and matrix spalling. In all these works, the combined effects of axial loading and bending of the inclined fiber are taken as superposition of those generated by fiber axial loading alone and fiber bending alone.

The present work examines the coupled effect of direct pullout load accompanied by bending load for fibers bridging a crack at an angle to the load direction. The analytic model is still based on the cantilevered beam representation of the fiber as in Morton and Groves (1974), but takes advantage of the knowledge of (matrix) foundation stiffness obtained from the numerical modeling of Leung and $\mathrm{Li}$ (1992). In addition, the phenomena of spalling of the brittle matrix below the fiber (the shaded area in Fig. 1), friction load concentration at the bending point, fiber debonding, and breakage are included in this model. An experimental study on the pullout mechanisms of inclined fiber was carried out to verify the model with experiment results.

As a side study, validity of the assumption of small beam deflection is examined. The results (Appendix I) show that there may be cases where large deflection of the fiber must be considered.

\section{ANALYTIC MODEL}

Morton and Groves (1974) proposed that an inclined fiber bridging a matrix crack (Fig. 1) can be divided into two parts at the midpoint of the crack by symmetry considerations. Each part of the fiber is then simulated as an elastic beam (Fig. 2), partially supported on a foundation (the matrix) and partially cantilevered out (crack bridging portion).

Geometric considerations require that the deflection $\delta$ and the cantilevered length $l$, be related to the crack opening $u$, the inclination angle $\theta$, and the fiber diameter $d$.

$$
\delta=0.5 u \sin \theta ; \quad l=0.5 d \tan \theta+0.5 u \cos \theta
$$

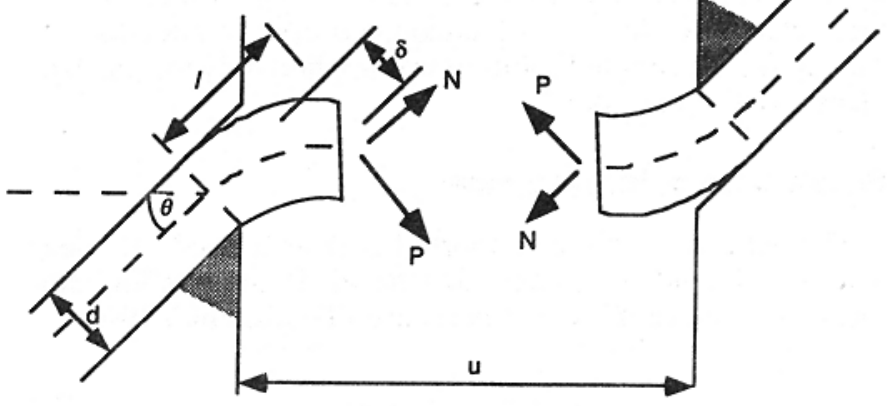

FIG. 1. Bending of An Inclined Fiber Bridging a Crack 


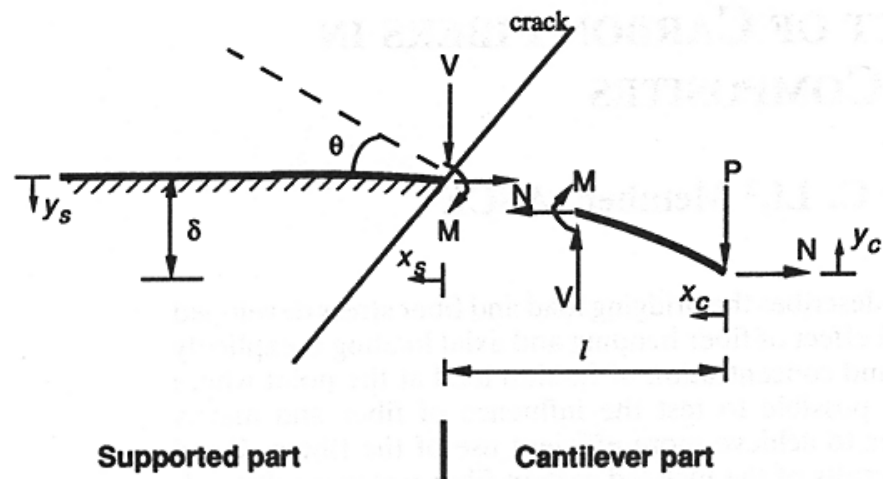

FIG. 2. Scheme of Loads for Supported and Cantilevered Parts of Fiber

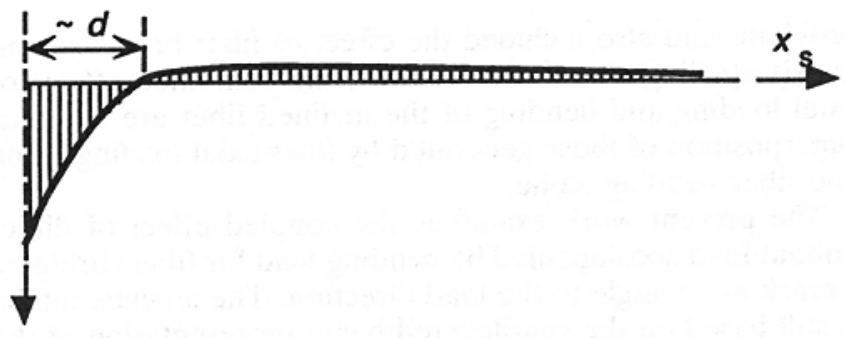

$y_{S}, R$

FIG. 3. Deflection and Reaction along Supported Part of Fiber

The analytical model was developed in the following manner: (1) Coupled equations based on the work of Hetenyi (1946) were obtained for the simple case of an inclined beam (fiber) on an elastic foundation subject to simultaneous bending load $P$ and axial load $N ;(2)$ the extent of matrix spalling under the fiber and its effect on the loads were determined; and (3) the effect of friction concentration at the point of fiber bending was included in the axial load.

With reference to Fig. 2, the cantilevered part of the beam is subjected to axial load, $N$ and bending load $P$. The supported part is subjected to the axial load $N$, shear load $V$, and bending moment $M$, transferred from the cantilevered part. Continuity considerations prescribe the boundary conditions for each part. The supported part can be considered a semi-infinite beam, since the loads and moment concentrate at the point where the fiber exits the matrix and decays rapidly into the embedded length of the fiber (Fig. 3), even for a short fiber length. For the sake of simplicity, the equations have been expressed with different $x-y$ axes for each part of the beam. In what follows, terms with the subscript $c$ refer to the cantilever part, and terms with a subscript $s$ refer to the supported part of the beam.

Coupling the solution for the supported part of the beam was found to have a negligible effect. For typical values of cementitious matrix and carbon fiber, the coupled equations of Hetenyi (1946) indicate a change of $2-4 \%$ in the bending load relative to that for the uncoupled case (Appendix II). Therefore, the coupled solution was applied only for the cantilever part of the fiber.

\section{Simple Case of Inclined Fiber}

The deflection of the supported part as a result of shear load $V$ and bending moment $M$ (the shear and bending moment from the cantilevered part) are (Timoshenko 1976)

$$
y_{s}=\frac{2 V \lambda}{k} e^{-\lambda x_{s}} \cos \left(\lambda x_{s}\right)
$$

$$
y_{s}=\frac{2 M \lambda^{2}}{k} e^{-\lambda x_{s}}\left[\cos \left(\lambda x_{s}\right)-\sin \left(\lambda x_{s}\right)\right]
$$

where $\lambda=\sqrt[4]{k / 4 E_{f} I_{f}} ; k=$ matrix foundation stiffness; and $E_{f}$ and $I_{f}=$ modulus of elasticity and moment of inertia of the fiber, respectively. The angle of slope, bending moment, and shear load of the fiber and the reaction from the matrix can be obtained as the first, second, third, and fourth derivatives of (3) and (4), respectively.

For the cantilevered part of the fiber, the equations were developed from the following, which describes the bending moment along the fiber developed from bending load $P$ and axial load $N$ acting at $x_{c}=0$ :

$$
M=-E_{f} I_{f} \frac{d^{2} y_{c}}{d x_{c}^{2}}=-N y_{c}+P x_{c}
$$

The solution to the differential (5), for the boundary conditions of $y_{c}=0$ at $x_{c}=0$ yields the equation for the deflection of the cantilivered part

$$
y_{c}=2 C \sinh \left(m x_{c}\right)+\frac{P}{N} x_{c}
$$

where $m=\sqrt{N / E_{f} I_{f}}$

Here, again, the deflected slope of the fiber, the moment, and shear load are the first, second, and third derivatives, respectively, of (6). Eq. (6) shows that increasing the axial load requires an increase in the bending load in order to maintain a constant fiber deflection. On the other hand, reducing the axial load increases the deflection to infinity as $N$ approaches 0 . In this case, the deflection becomes too large and the theory of large deflection must be applied (Appendix I). In the case of fiber bridging a crack, axial and bending loads are developed simultaneously, and the risk of very small axial load relative to the bending load does not exist.

From continuity considerations, the angle of slope of the fiber, $\phi$, at the edge of the supported part $\left(x_{s}=0\right)$ has to be equal to the angle of the cantilever part at $x_{c}=l$. The total deflection of the two parts is the deflection $\delta$ needed to deflect the fiber to the midpoint of the crack, as was expressed in (1). These conditions are represented by

$$
\phi_{s}\left(x_{s}=0\right)=\phi_{c}\left(x_{c}=l\right) ; \quad y_{s}\left(x_{s}=0\right)+y_{c}\left(x_{c}=l\right)=\delta
$$

The unknown moment $M$, shear force $V$, and integration constant $C$ can be obtained by enforcing these two conditions, along with moment continuity. As a result, the total deflection can be expressed as

$$
\delta=K_{2} P
$$

where

$K_{2}=-\frac{4 \lambda}{k} K_{1} N[m \cosh (m l)+\lambda \sinh (m l)]+2 K_{1} \sinh (m l)+\frac{l}{N}$

$\frac{1}{K_{1}}=-\frac{4 \lambda^{2} N^{2}}{k}[m \cosh (m l)+\lambda \sinh (m l)]-2 N m \cosh (m l)$

The axial load $N$ is calculated according to (12) ( $\mathrm{Li} \mathrm{1992),}$ assuming that the fiber-matrix bond, $\tau$, is of friction nature only. When considering the axial load, the pullout length $l_{N}$ should be only the second term in (2), $(0.5 u \cos \theta)$, as the other term in (2) is related to the part of the fiber that separates from the matrix on bending (as shown in Fig. 1). 


$$
N\left(l_{N}\right)= \begin{cases}\pi \sqrt{\frac{l}{2} E_{f} d^{3} \tau l_{N}} & l_{N}<l_{o} \\ \pi \tau L d\left(1-\frac{l_{N}-l_{o}}{L}\right) & l_{o}<l_{N}<L \\ 0 & l_{N}>L\end{cases}
$$

where $l_{o}=2 L^{2} \tau / E_{f} d ; l_{N}=0.5 u \cos \theta$; and $L=$ fiber embedment length.

Special attention must be given to the parameter of the matrix stiffness $k$ used in the model. This parameter refers to the matrix deformation under a unit load applied over a unit length of fiber. This parameter is related to the matrix modulus of elasticity $E_{m}$, but also takes into account the foundation depth variation with the distance along the embedded fiber length from the matrix crack (see Fig. 1). This threedimensional problem was analyzed using the finite-element method by Leung and $\mathrm{Li}$ (1992), who found that the ratio $k / E_{m}$ is approximately 0.20 close to the crack and increases to about 0.45 far from the crack into the matrix, for the case of equal fiber and matrix moduli, i.e., $E_{f} / E_{m}=1$. The ratio $k / E_{m}$ at any point along the fiber increases slightly for fibers stiffer than the matrix, and does not change much for $E_{f} / E_{m}$ $>6$. For a high fiber modulus, $k / E_{m}$ was determined to be 0.23 near the crack and equals 0.55 far from the crack. As the load concentration in the fiber and the matrix is located along a short length of the fiber from the crack into the matrix (Fig. 3), an average value of $k / E_{m}=0.25$ will be used in the following discussion.

There are no experimental values on the stiffness of the matrix as defined in this case to validate the analytical model, and it is reasonable to assume that this property must be determined in a region close to the fiber. This transition-zone region has been shown to be different from the bulk matrix in microstructure and properties (Wei et al. 1986; Bentur et al. 1985). The effect of changes in the matrix properties will be discussed later.

\section{Matrix Spalling}

After solving (9)-(12), all loads and moments can be calculated. Introducing these loads and moments into the fourth derivatives of (3) and (4) yields the reaction per unit length from the matrix to the fiber. This reaction, divided by the fiber diameter, also expresses the pressure of the fiber on the matrix. Adopting the assumption of Leung and Li (1992), matrix spalling occurs when this pressure exceeds the matrix ultimate compressive spalling strength $\sigma_{m}=\varepsilon_{m} E_{m}$. The compressive spalling strength may be higher than the bulk compressive strength of the matrix because of the small sampling volume on which the fiber pressure load is applied. The distance from the crack to the point at which the matrix reaches its ultimate strength is the spall length. This length is added to the free length of the fiber, calculated in (2), and the loads and moments are calculated again for the new geometric conditions. This procedure was repeated until satisfactory convergence was achieved.

\section{Friction Concentration}

The concentration of normal load from the matrix toward the fiber at the bending point leads to a concentration of friction load, $N_{f}$, in this region, which increases the axial load in the fiber. This load can be calculated by integrating the reaction of the matrix along the fiber $R$ and multiplying it by the friction coefficient $f$

$$
N_{f}=f R
$$

As the reaction of the fiber is wavy in shape and decays rapidly along the fiber (Fig. 3), only the first wave was considered. The rest of the reaction load along the fiber was ignored.

The friction effect is calculated by adding the friction load $N_{f}$, calculated from (13), to the axial load due to pullout (12). Increasing the axial load $(N)$ requires an increase in the bending load $(P)$, to maintain a constant deflection $\delta$, resulting in an increase of the matrix reaction force and spalling length. However, the effect of changes in the axial and bending loads on the spalling length are very small and, after one iteration, satisfactory convergence was achieved.

\section{Determination of Final Bridging Load and Fiber Stress}

After considering the spalling effect and friction effect, the fiber bridging load is calculated according to

$$
F_{\text {brdg }}=P \sin \theta+\left(N+N_{f}\right) \cos \theta
$$

The maximum tensile stress in the fiber due to pullout and bending was calculated at the point where the fiber exits the matrix, to determine whether fiber breakage occurs. The actual point of maximum stress due to bending alone is located a short distance in the matrix. However, the axial load is reduced along this distance due to the friction effect, which compensates for the increase in the bending stress. The change of the real maximum stress from the stress calculated at the exit point is very small (less than 5\%) and was ignored. The bending moment at the point the fiber exits the matrix is

$$
M=-2 K_{1} P N \sinh (m l)
$$

and the maximum tensile stress in the fiber at this point is

$$
\sigma=\sigma_{b}+\sigma_{p}=\frac{M d}{2 I_{f}}+\frac{4\left(N+N_{f}\right)}{\pi d^{2}}
$$

\section{IMPLICATIONS OF ANALYTIC MODEL}

The need for the new model was tested by comparing the bending stress developed in the fiber for cases of the coupled and uncoupled solutions of the bending and axial loads. The main effect of coupling the solution is on the cantilevered part of the fiber, as the deflection of the supported part is restrained by the matrix. Therefore, the development of bending moment at a perfectly (no spalling) cantilevered beam of various lengths (as the crack opens) was compared for the two cases.

For this calculation, a carbon fiber with typical geometric and mechanical properties is assumed. The axial stress in the fiber were calculated using (12). The deflection of the cantilevered beam and its cantilevered length were calculated according to (1) and (2), respectively. The stress for the coupled solution was calculated by solving (6) for boundary conditions $\phi_{c}\left(x_{c}=l\right)=0$ and $y_{c}\left(x_{c}=l\right)=\delta$, and for the uncoupled case by using simple elastic theory $\left[\delta=P l^{3} /\left(E_{f} I_{f}\right)\right]$. These solutions are schematically presented in Fig. 4. The calculation as presented does not take into account the possibility of fiber breakage, matrix deformation, or spalling.

For the uncoupled solution, it is seen that for a small crack width the bending stress increases rapidly, faster than the stress developed by the coupled solution. However, as the crack extends, the bending stress calculated by the uncoupled solution reduces while the stress calculated by the coupled solution continues to increase. This phenomenon can be explained by the nature of the coupled solution. The axial load induces a moment opposing the direction of the moment coming from the bending load, leading to a lower bending moment, relative to the case of the uncoupled solution. However, as the axial load increases for an increase in crack width, the bending load is also increased to maintain the deflection $\delta$ [see (6)], leading to a continuous increase in the bending 


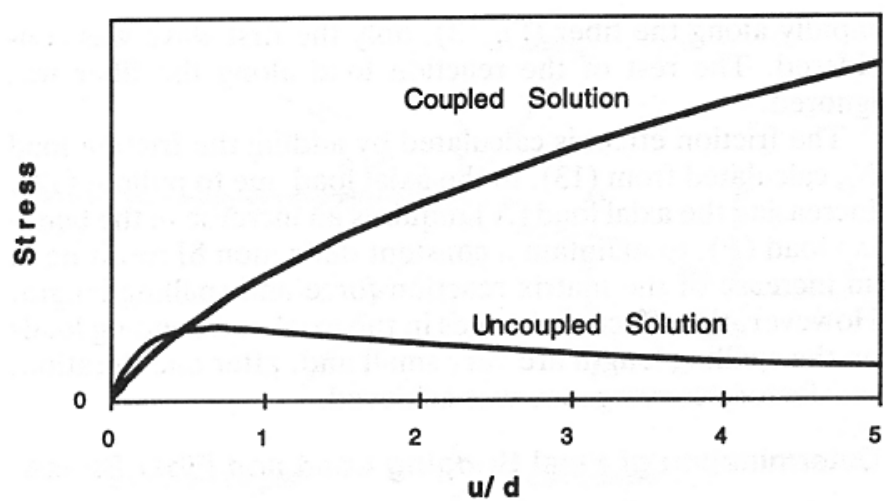

FIG. 4. Comparison between Coupled and Uncoupled Solutions for Fiber Stress Development as Function of Crack Width

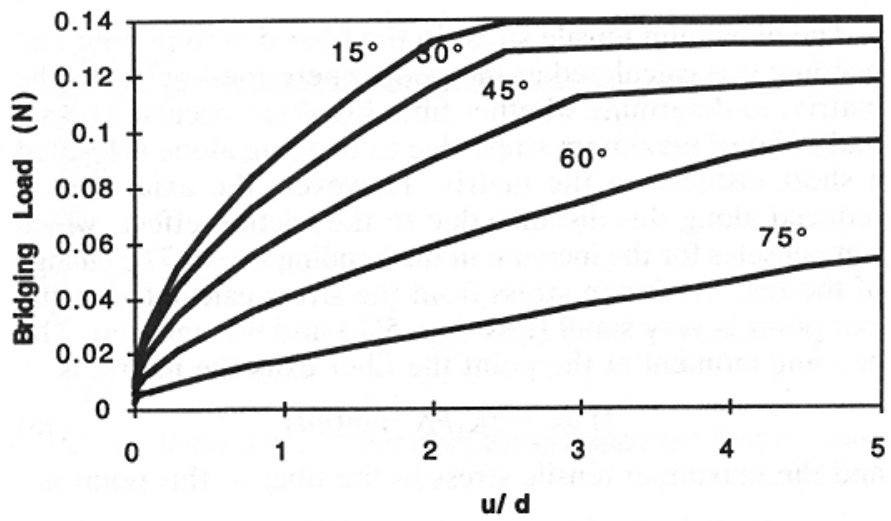

FIG. 5. Bridging Load versus Crack Width for Different Inclination Angles

moment. For the uncoupled solution, the bending load reaches a maximum at the short length of the cantilevered fiber (small crack width) but reduces for a longer length, leading to a reduction in the bending moment and fiber stress.

The foregoing analysis shows that using the uncoupled solution may lead to overestimation of the bending stress and contribution of the bending load to the total bridging load for small crack width, and to a severe underestimation of these parameters for a large crack width. However, this difference should diminish if matrix spalling occurs, since the free length of the fiber and the moment arm for the bending force increases, while the moment arm for the axial load essentially stays the same. Therefore, a somewhat less pronounced coupled effect results. Solutions for a large crack width may be cut off if the fiber breaks in bending. Fig. 4 should be understood with these considerations in mind.

Typical carbon fiber and cement matrix parameters were introduced into the equations of the complete coupled solution [(9)-(12)] for the discussion in the next section. The following parameters were kept constant while the effect of changing the other parameters was studied: fiber diameter $d$ $=15 \mu \mathrm{m}, L=3 \mathrm{~mm}, k / E_{m}=0.25, \varepsilon_{m}=0.2 \%, \tau=1 \mathrm{MPa}$, and $f=0.5$.

In general, both the bridging load and the fiber stress increase as the crack opens [for $l_{N}<l_{0}$ defined in (12)], as can be seen in Figs. 5 and 6 , respectively. The bridging load decreases as the angle increases, as shown in Fig. 5, mainly due to the effect of the angle on the axial load $[N \cos \theta$ in (14)], which decreases as the angle increases. The bending load is low, relative to the axial load, and does not affect the bridging load much, although its component in (14) increases with the angle. This load, however, has a strong effect on the bending stress, which increases as the angle increases. Generally, the fiber stress increases with the angle for a given

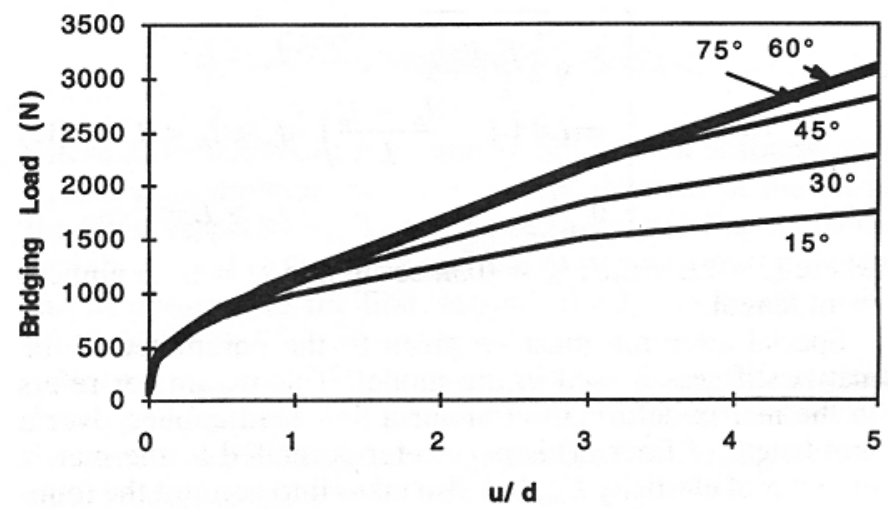

FIG. 6. Fiber Tensile Stress versus Crack Width for Different Inclination Angles

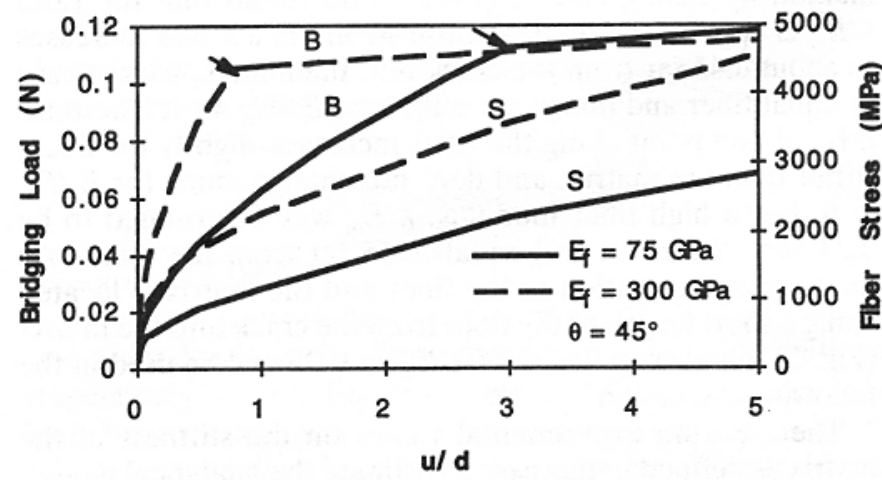

FIG. 7. Effect of Fiber Modulus on Bridging Load and Fiber Stress as Crack Opens

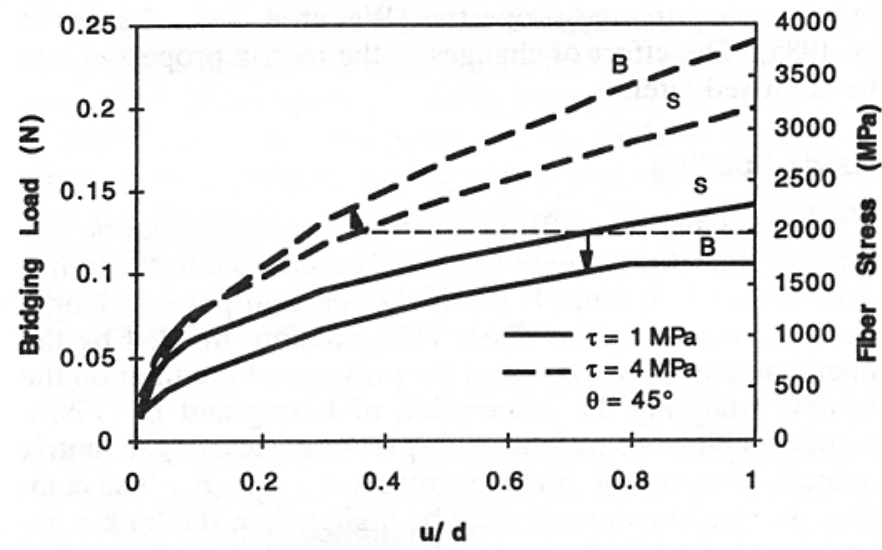

FIG. 8. Effect of Bond Strength on Bridging Load and Fiber Stress as Crack Opens

crack width. However, the bending load is affected by many parameters, such as the axial load it needs to balance to maintain the deflection $\delta[(6)]$, spalling length, and other geometrical and material parameters. Therefore, there may be cases where fiber stress does not increase with the angle or decreases slightly, as shown in Fig. 6 for $75^{\circ}$.

The effect of changes in the fiber modulus of elasticity is presented in Fig. 7, for fiber inclined at $45^{\circ}$. A low modulus of $75 \mathrm{GPa}$ is represented by the solid line and a high modulus of $300 \mathrm{GPa}$ is represented by the dashed line. The matrix modulus of elasticity is $30 \mathrm{GPa}$ and the compressive strength is $60 \mathrm{MPa}$. For convenience, both the bridging load and the fiber tensile stress were plotted on the same chart. The contribution of fiber bending to the bridging load can be clearly seen on the curves of the bridging load. For the case of pullout only, this curve is of descending portion after the pullout load 


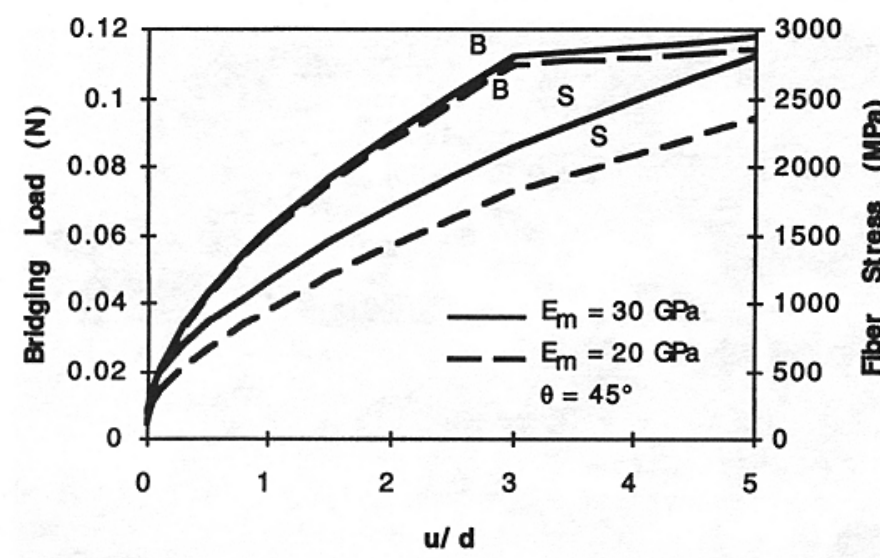

FIG. 9. Effect of Matrix Properties on Bridging Load and Fiber Stress as Crack Opens

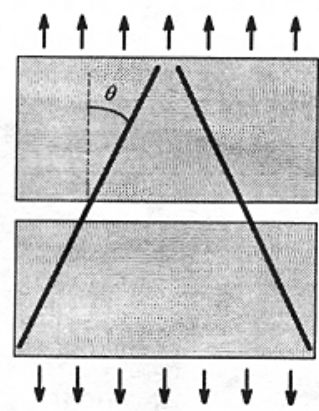

FIG. 10. Test Setup of Inclined Fibers

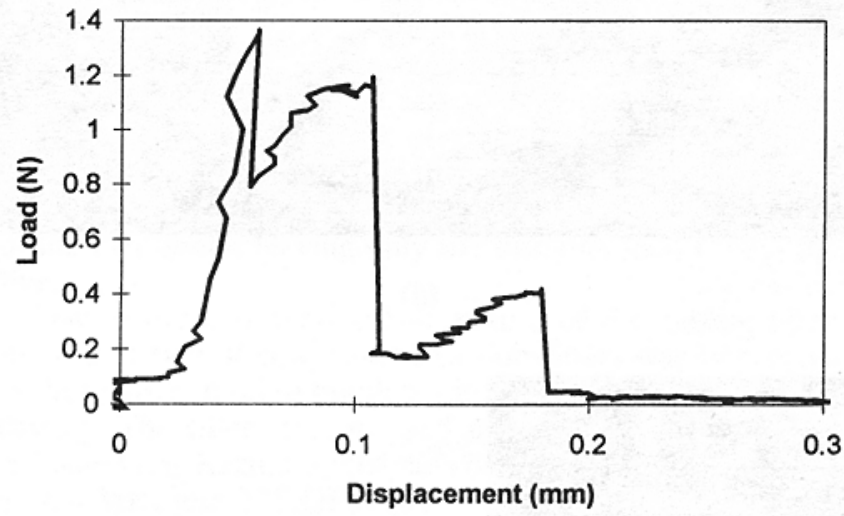

FIG. 11. Typical Load-Displacement Curve of Two-Part Specimen Test (Fiber Angle $=45^{\circ}$ )

reaches its peak, marked by arrows on the curves of the bending load in Fig. 7. By including the bending effect, a slight increase can be seen in the region after the pullout peak, resulting from a continuous increase in the bending load. Fiber stress increases more rapidly for the high modulus fiber because of the increase in bending rigidity, leading to possible failure of the fiber at the smaller crack width, for a given strength. This analysis is valid for fibers of the same strength capacity. However, a lower modulus is generally accompanied by a lower tensile stress capacity; therefore, low modulus fiber may break before reaching the point where the higher bridging stress develops, so each case is dealt with separately.

Fig. 8 represents the effect of change in bond strength on fiber stress and bridging load, computed for high modulus fibers $\left(E_{f}=300 \mathrm{GPa}\right)$. Two bond values were assumed: 1
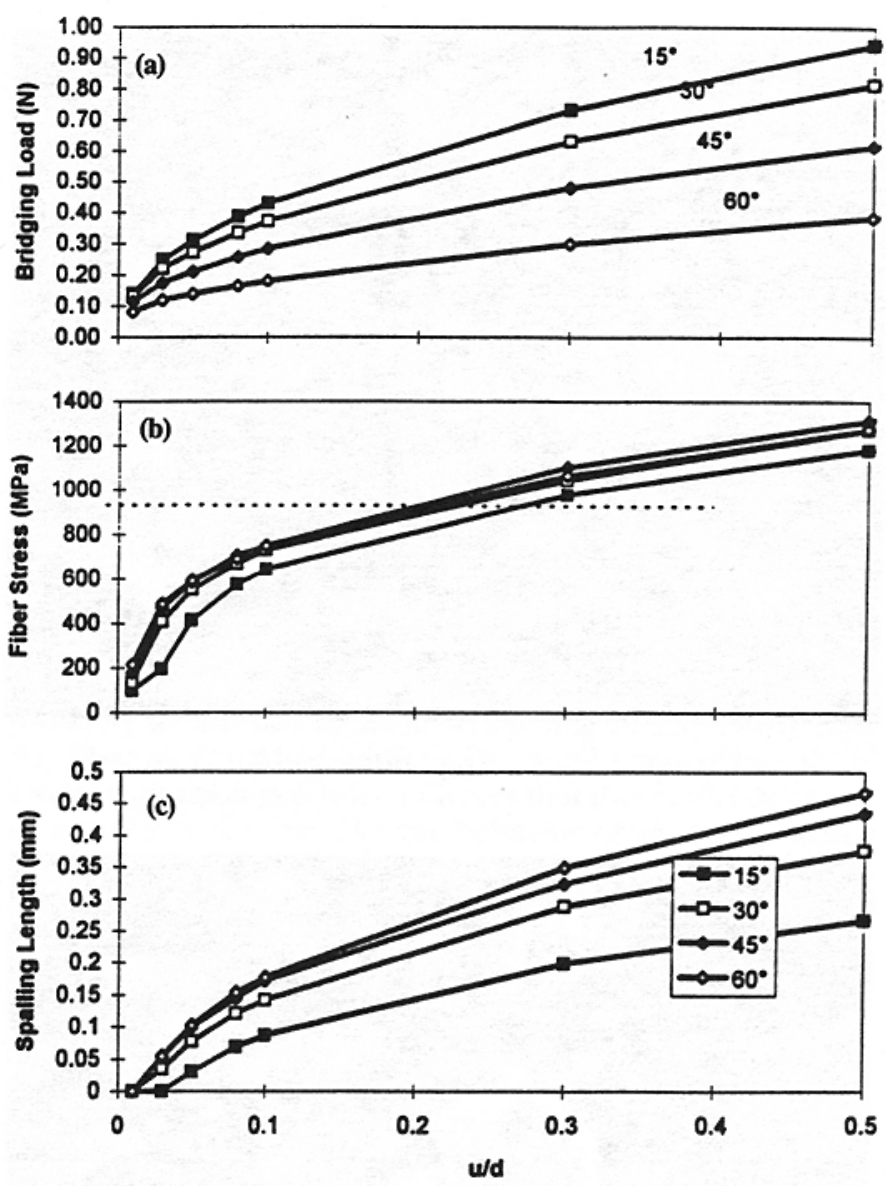

FIG. 12. Model Prediction of: (a) Bridging Load; (b) Fiber Stress; (c) Spalling Length for Tested System

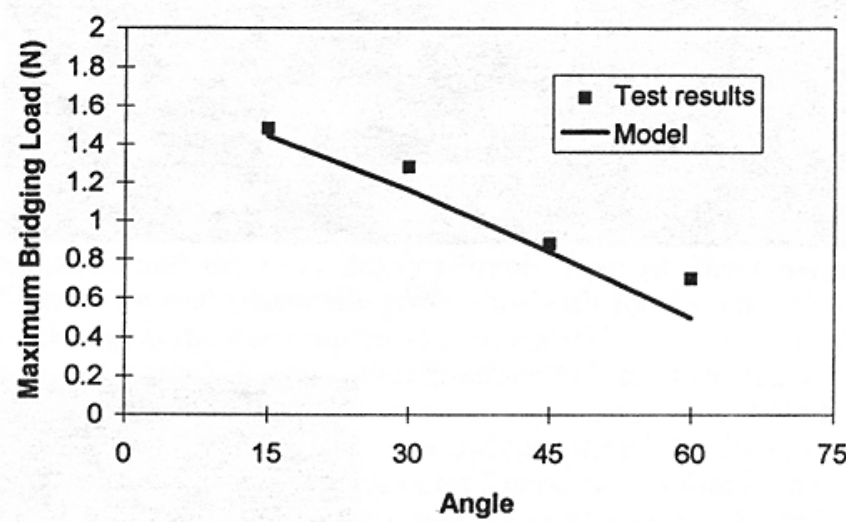

FIG. 13. Effect of Inclination Angle on Maximum Bridging Load Prior to Fiber Breakage; Experimental Results and Analytic Model Prediction

$\mathrm{MPa}$ is represented by the solid line, and $4 \mathrm{MPa}$ is represented by the dashed line. Here too, the pullout resistance has the strongest effect on the bridging load, while both pullout and bending resistance affect the fiber stress. Although the higher bond strength leads to a remarkable increase in the bridging load for a given crack width, fiber breakage due to high fiber stress will occur at a smaller crack width, leading to a smaller effect of the improved bond. Assuming a fiber tensile strength of 2,000 MPa (represented by the thin dotted line in Fig. 8), the critical crack width for fiber breakage is approximately 0.8 and 0.4 fiber diameters for bond strengths of 1 and $4 \mathrm{MPa}$, respectively. As the critical crack width is reduced when the bond is increased, the full potential of increasing the bond is not utilized, as indicated in the figure.

The change in matrix properties affects the bridging load 


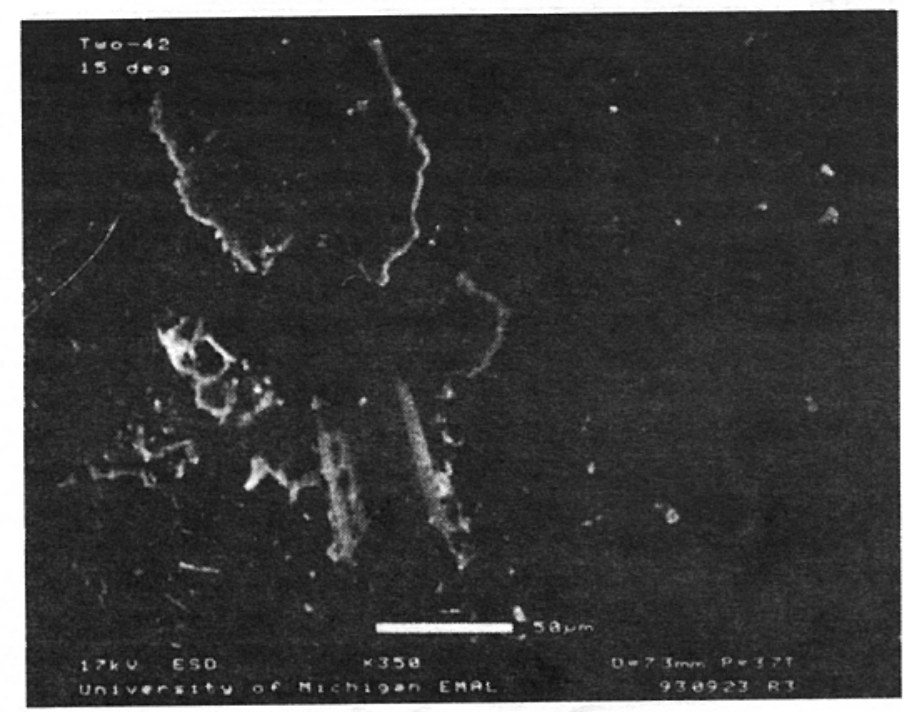

(a)

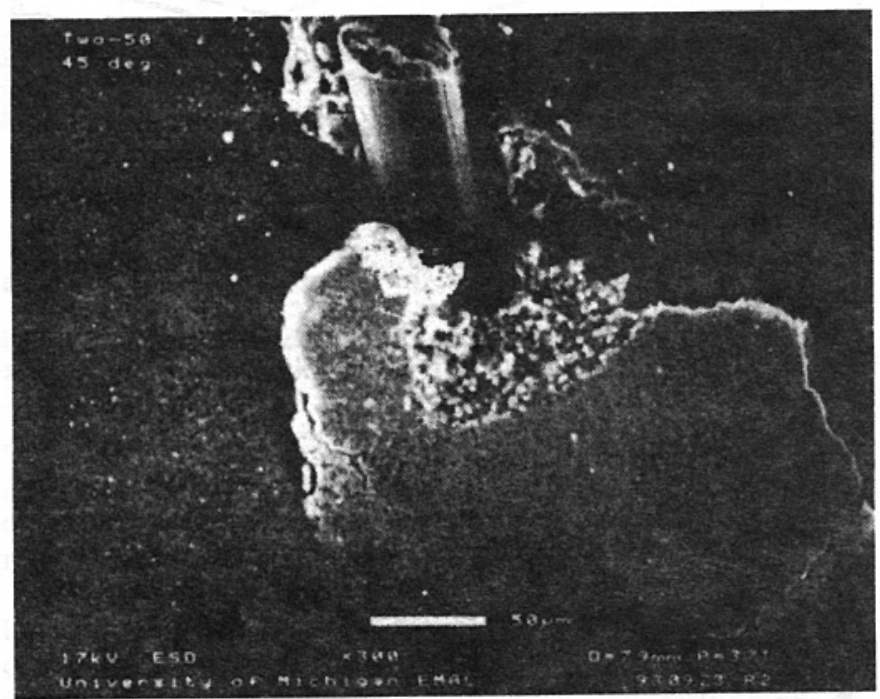

(c)

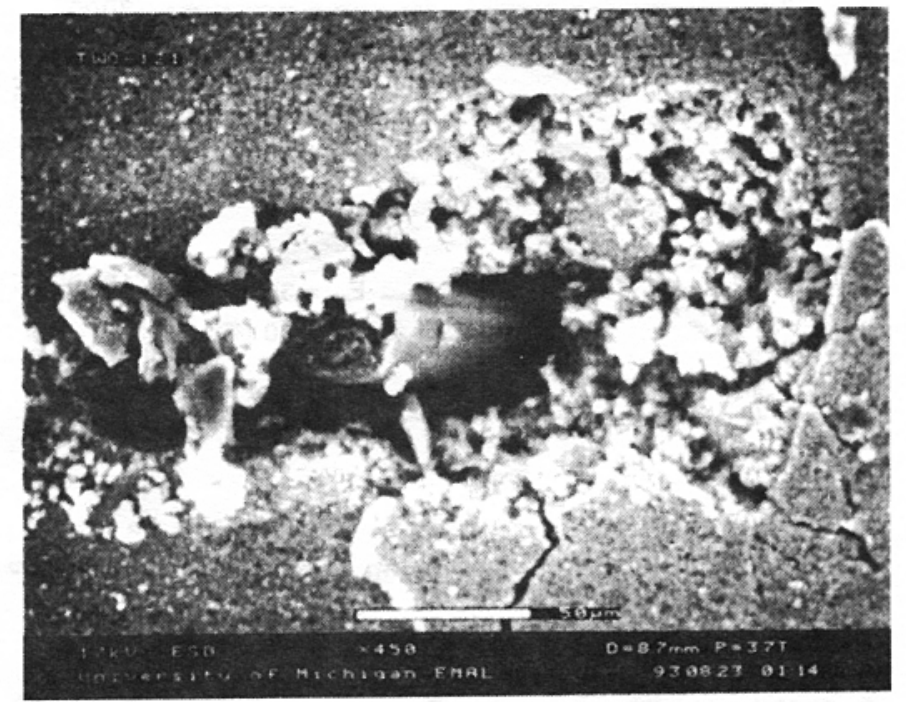

(b)

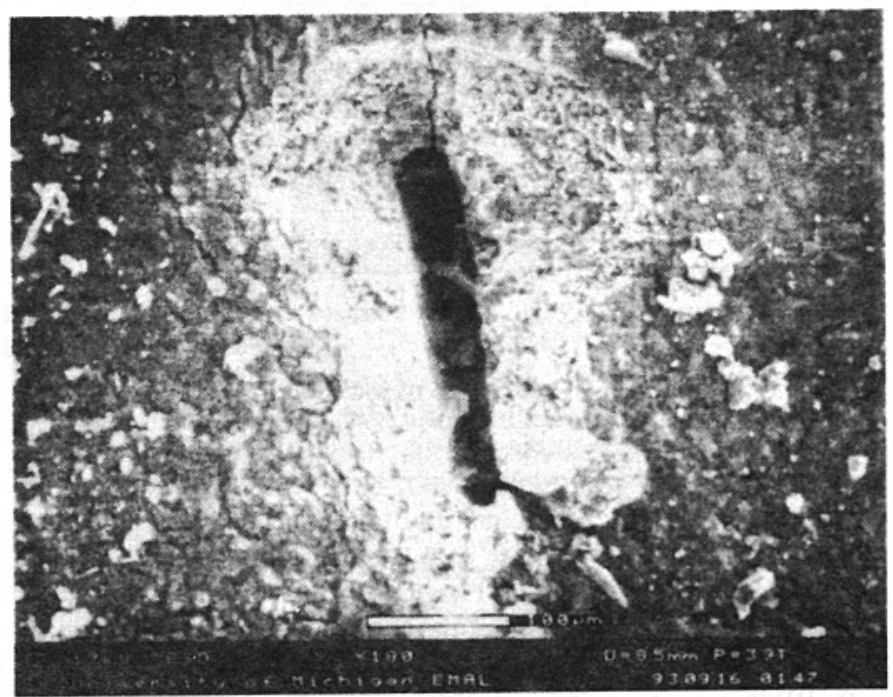

(d)

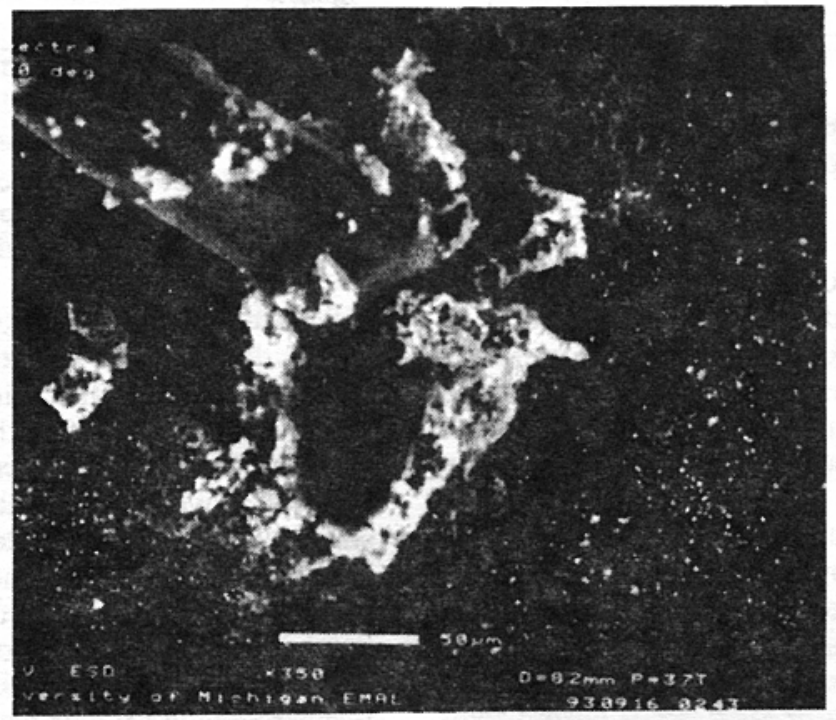

(e)

FIG. 14. ESEM Micrographs of Specimens for Fibers Inclined at: (a) $15^{\circ}$; (b) $30^{\circ}$; (c) $45^{\circ}$; (d) $60^{\circ}$; (e) Ductile Fiber Incline at $30^{\circ}$ 
and fiber stress in two ways: (1) Increase of the matrix stiffness $k$ reduces matrix deformation and increases the bending stress; and (2) generally, an increase in the matrix modulus is accompanied by an increase in the strength of the matrix, which may change the spalling length of the matrix, leading to an additional effect on the fiber stress development. A relation of $E \propto \sqrt{\sigma}$ is generally accepted for cementitious materials (Soroka 1979).

For the example in Fig. 9, matrix modulus of $30 \mathrm{GPa}$ and strength of $60 \mathrm{MPa}$ were compared with matrix modulus of $20 \mathrm{GPa}$ and strength of $27 \mathrm{MPa}$, for low modulus fibers $\left(E_{f}\right.$ $=75 \mathrm{GPa}$ ). Fig. 9 shows that changing the matrix properties does not change the bridging load significantly, as this load is mainly affected by the pullout resistance, which is not influenced by the matrix properties but more by the fiber and bond properties. However, based on experimental studies by Katz and $\mathrm{Li}$ (1994), it is reasonable to assume that increasing the matrix properties will also improve the bond strength. The main effect of changing the matrix properties is on the bending stress that develops in the fiber. Fig. 9 shows that a fiber embedded in a weak and low modulus matrix suffers lower tensile stress than fiber embedded in a strong matrix. Thus, the fiber can survive at a larger crack width and can support more bridging loads.

This conclusion stands in good agreement with the results of Katz and Bentur (1994), which observed strength reduction in carbon fiber-reinforced cement composites when the matrix became denser by the effect of time or by using silica fume.

\section{EXPERIMENTAL STUDY}

A limited experimental study was carried out using an experimental thick carbon fiber $(d=46 \mu \mathrm{m})$ prepared by Conoco Inc., Ponca City, Oklahoma. The setup consisted of two fibers arranged at an angle $\theta$ to the load direction in a cement matrix, as shown in Fig. 10. Each part of the cement matrix was cast separately on subsequent days and a thin layer of oil was applied on the first mix prior to the second cast, in order to minimize the bond between the two parts. The specimen was glued to the testing machine and direct tension was applied. This setup provides balance of the horizontal loads of the two fibers, leaving only the bridging load to be effective.

Due to the extremely brittle nature of the carbon fibers, the use of typical commercial carbon fibers was impractical as these fibers tend to break while setting the mold or during casting. The fiber strength and modulus of elasticity were estimated (A. Kazmer, personal communications, June, 1994) as $930 \mathrm{MPa}$ and $175 \mathrm{GPa}$, respectively. The cement matrix was Type III cement paste, with a water to cement ratio of 0.5 . Specimens were kept in a humid environment until testing day, seven days after the second cast. Test results are based on at least five specimens.

The inclination angle was $15^{\circ}, 30^{\circ}, 45^{\circ}, 60^{\circ}$, and $75^{\circ}$. However, the formation of the cement matrix around the fibers at high angles was not perfect, especially for the $75^{\circ}$ specimen, because of the very thin cement layer above the fibers in the half-specimen cast first. This layer, which affects the fiber bending stress, is strongly influenced by bleeding, shrinkage, and other weaknesses of the cement paste. Therefore, results of the $75^{\circ}$ specimens are not included here.

A typical load-displacement curve for fibers inclined at $45^{\circ}$ is presented in Fig. 11. The first peak in the curve is associated with the remaining bond between the two parts of the matrix specimen. The second peak is the maximum bridging load when one of the two fibers breaks. The other fiber breaks subsequently at a lower load, reflected by the third low peak in the test curve.
To simulate these inclined bridging fiber experiments, fiber data along with the matrix properties of $E_{m}=30 \mathrm{GPa}$ and $\sigma_{m}=60 \mathrm{MPa}$ and bond strength of $0.5 \mathrm{MPa}$, were introduced into the model described earlier. The results are presented in Fig. 12(a) for the bridging load, Fig. 12(b) for the fiber stress, and Fig. 12(c) for the spalling length. The horizontal dotted line in 12(b) represents the fiber strength as measured for this fiber. At this stress the fiber breaks; this corresponds to $u / d=0.20-0.28$, as shown in Fig. 12(b). The maximum bridging load in the tests was predicted by determining the bridging load at the crack width corresponding to fiber breakage [Fig. 12(a)]. For $30^{\circ}$, as an example, the model predicts a bridging load of $\sim 0.58 \mathrm{~N}$ for a single fiber, which is close to half the measured value $(1.28 \mathrm{~N})$ for two fibers tested in the experimental work.

Additional comparison of test results with the analytical model for other inclination angles is presented in Fig. 13. The results show very good agreement with the model. The predicted values are slightly lower than the experimental results, probably as a result of some weakening of the matrix near the fiber as described earlier. The good comparison of the model with the test results indicates that this model can serve as a useful tool to predict the behavior of random-oriented brittle fibers in cementitious composites.

Spalling of the matrix, which affects the bending stress in the fiber, can be seen in Fig. 14. These photographs were taken by an environmental scanning electron microscope (ESEM) from the surface of the lower specimen, and show fibers inclined at $15^{\circ}$ through $60^{\circ}$. The mode and extent of damage to the matrix appears different for the different angles. At $15^{\circ}$, the extent of damage looks small and seems more like local crushing of the matrix. For the $60^{\circ}$ case, the damage extends for a longer distance and appears more like shear failure of the matrix. This indicates that better modeling of the matrix is needed in order to calculate the stress in the fiber correctly.

The differences between the action of brittle fiber and ductile fiber in bridging a crack can be seen by comparing Figs. 14(b) and 14(e). Fig. 14(b) shows brittle carbon fiber after the test, and Fig. 14(e) shows ductile fiber (spectra, a high modulus polyethylene fiber with similar diameter). The extent of damage to the matrix in the ductile fiber was more localized and the fiber did not break. Instead, the polymer fiber deformed plastically (probably with local compressive buckling) at the exit point, and continued to support the loads across the crack. On the other hand, carbon fiber caused more extensive damage to the surrounding paste, and the fiber broke down when it reached its ultimate strength. These contrasts explain why, for polymeric fibers, the snubbing model described by Li et al. (1990), which predicts increasing bridging load with higher inclined angles, works so well. However, for brittle fibers like most carbon fibers, the extensive local matrix crushing and fiber bending failure may cause a bridging load reduction with an inclination angle, as revealed by the experiments reported here and predicted by the aforementioned model.

The spalling length was estimated based on the observations of the specimen surface in Fig. 14, giving a spalling length of approximately 4-6 fiber diameters for angle changes from $15^{\circ}$ to $60^{\circ}$, respectively. These results also stand in good agreement with the model predictions represented in Fig. 12 (c), qualitatively verifying the validity of the model.

\section{CONCLUSIONS}

An analytic model was developed to describe the bridging load and fiber stress in an inclined brittle fiber bridging a matrix crack, for different fiber and matrix parameters. Comparison of the coupled solution of bending and axial load with 
the uncoupled solution suggests the possibility of overestimation and underestimation of the bending stress for small and large crack widths, respectively. However, the difference between the coupled and uncoupled solution at a large crack width is tempered by the matrix spalling and fiber breakage. At any rate, a more accurate account of the fiber stress and bridging load for a brittle fiber bridging across a brittle matrix crack was offered by the coupled model described in this paper.

The bridging load was found to be affected mainly by the development of the axial load from fiber pullout. Increase in the fiber modulus or fiber-matrix bond strength results in a higher bridging load for a given crack width. The bending stress, which leads to fiber rupture in brittle fibers, is affected by the fiber modulus and fiber-matrix bond strength along with the effect of matrix parameters. An increase in the fiber modulus, bond strength, or matrix modulus raised the fiber stress for a given crack width, and resulted in fiber failure at a smaller crack width for a given fiber strength, leading to a lower bridging load than expected from the improvement in these material properties.

The model was compared with an experimental study of inclined large diameter carbon fibers in a cement matrix. Reasonable comparisons of model predictions with experimental results, both for the bridging load and the matrix spall length, were achieved. Therefore, the model can serve as a useful tool to analyze the efficiency of inclined brittle fibers in composite postcracking properties.

\section{ACKNOWLEDGMENTS}

This research was partially funded by a grant from Conoco Inc., Ponca City, Oklahoma, to the Advanced Civil Engineering Materials Research Laboratory (ACE-MRL) at the University of Michigan. Special thanks are due to Dr. McConaghy for many helpful discussions and to Dr. A. J. Kazmer who made the experimental fiber available for this research. Useful comments by Dr. C. Leung are also acknowledged.

\section{APPENDIX I. LARGE DEFLECTION}

\section{,}

As shown in Fig. 1, the deflection of the fiber may be large. The small deflection theory may not be applicable to the solution when the deflection is large, and will result in an overestimation of the bending loads. In this case, a large deflection must be considered. This problem is more likely to occur in the cantilevered part of the fiber, as the deflection of the supported part is restricted by the matrix. Therefore, in the following we only treat the case of large deflection in the cantilever part of the beam.

For large deflection of a cantilever beam, the exact solution of the equation of the deflection curve was solved by Gare and Timoshenko (1984). The exact relation between the bending load to the vertical deflection of the fiber end, $\delta_{v}$, is expressed as

$$
\frac{\delta_{v}}{L}=1-\sqrt{\frac{4 E_{f} I_{f}}{P L^{2}}}[E(g)-E(g, \alpha)]
$$

where $E_{f}=$ modulus of elasticity of the fiber; $I_{f}=$ moment of inertia of the fiber; $E(g)=$ complete elliptic integral of the second type; $E(g, \alpha)=$ incomplete integral of the second type; $g=\sqrt{(1+\sin \phi) / 2} ; \alpha=\arcsin 1 /(g \sqrt{2}) ;$ and $\phi=$ angle of slope of the fiber.

The solution to this equation is presented in Fig. 15 along with the solution for small deflection.

Fig. 15 shows that large deflection of the fiber may lead to a severe error in the calculations based on the small deflection theory. There is a difference of $10 \%$ for the calculated deflection according to the two methods at $P l^{2} / E I \cong 1.0$. This value was introduced to the small deflection model with typ-

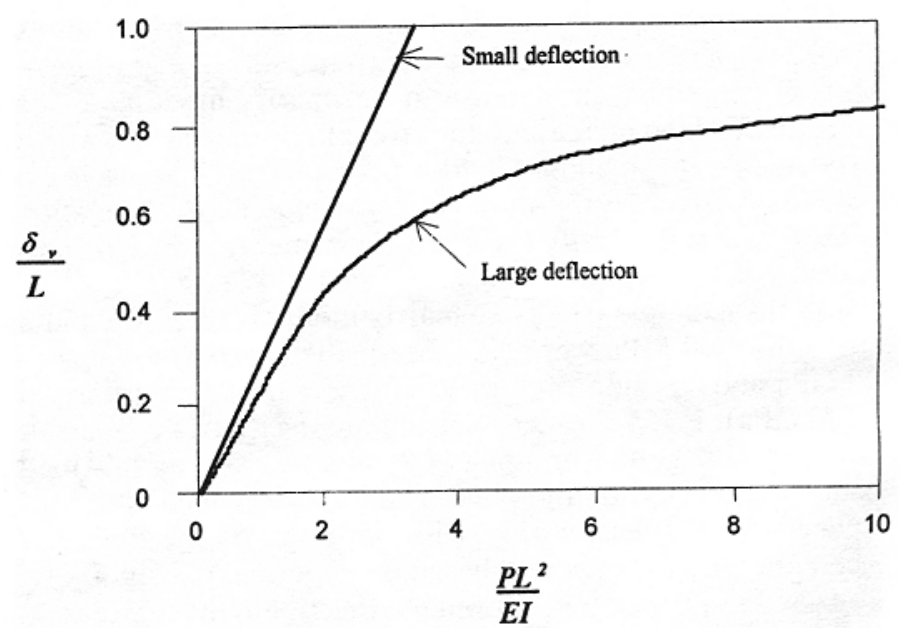

FIG. 15. Predicted Vertical Deflection of Cantilever Beam Subjected to Concentrated Load at End, Based on Small- and LargeDeflection Theory [after Gare and Timoshenko (1984)]

ical fiber parameters. For high modulus carbon fibers, such as the typical pan type, the critical crack width at which large deflection must be considered is 8,6 , and 5 fiber diameters for fibers inclined at $45^{\circ}, 60^{\circ}$, and $75^{\circ}$, respectively. For low modulus fiber, such as the pitch type, the crack width is 5 , 4 , and 3.7 fiber diameters for $45^{\circ}, 60^{\circ}$, and $75^{\circ}$, respectively. It seems that as the crack opens beyond these limits, the deflection of the fiber is considered large. When calculating the bending stress for these regions of crack width, the theory of large deflection may be needed.

\section{APPENDIX II. COUPLED SOLUTION FOR SUPPORTED PART OF FIBER}

For a beam laid on a continuous foundation subjected to axial load $N$, bending load $P$, or bending moment $M$, the following were proposed by Hetenyi (1946) for the deflection line

$$
\begin{aligned}
y_{s}(P, N) & =\frac{P}{\beta k} \frac{2 \lambda^{2}}{\left(3 \alpha^{2}-\beta^{2}\right)} e^{-\alpha x_{s}}\left[2 \alpha \beta \cos \left(\beta x_{s}\right)\right. \\
\left.+\left(\alpha^{2}-\beta^{2}\right) \sin \left(\beta x_{s}\right)\right] & \\
y_{s}(M, N) & =\frac{M}{E_{f} I_{f}} \frac{1}{\left(3 \alpha^{2}-\beta^{2}\right)} \frac{1}{\beta} e^{-\alpha x_{s}}\left[\beta \cos \left(\beta x_{s}\right)+\alpha \sin \left(\beta x_{s}\right)\right]
\end{aligned}
$$

where $\lambda=\sqrt[4]{k / 4 E_{f} I_{f}} ; \alpha=\sqrt{\sqrt{k / 4 E_{f} I_{f}}+N / 4 E_{f} I_{f}} ; \beta=$ $\sqrt{\sqrt{k / 4 E_{f} I_{f}}-N / 4 E_{f} I_{f}}$; and $k=$ foundation stiffness.

If the axial load is small relative to the fiber stiffness, $\alpha \approx$ $\beta$, and the equations assume the form as in the bending case only, regardless of the axial load effect. This occurs when

$$
\frac{N}{4 E_{f} I_{f}} \ll \sqrt{\frac{k}{4 E_{f} I_{f}}}
$$

The maximum axial load that can be developed in the fiber is defined by the fiber ultimate strength, $N=\sigma_{f} \pi d^{2} / 4$, and (20) becomes

$$
1<\sqrt{\frac{k E_{f}}{\pi \sigma_{f}^{2}}}
$$

Introducing typical values of pan- and pitch-type carbon fibers $\left(E_{f}=300\right.$ and $50 \mathrm{GPa}$ and $\sigma_{f}=3,000$ and $700 \mathrm{MPa}$, respectively) into (21) yields a value of 10 and 18 , respectively, for the right-hand side of (21). These values underestimate the real values as the axial load never reaches the 
ultimate strength of the fiber because of the bending loads, which develop simultaneously. The results indicate that for commonly used carbon fibers, the calculation can be done neglecting the effect of the coupled solution on the supported part of the fiber.

\section{APPENDIX III. REFERENCES}

Allen, H. G. (1972). "The strength of thin composites of finite width with brittle matrices and random discontinuous reinforcing fibers." $J$. Phys. D: Appl. Phys., 5(2), 331-343.

Aveston, J., Mercer, R. A., and Sillwood, J. M. (1974). "Fiber reinforced cements - scientific foundations for specifications." Proc., Nat. Phys. Lab. Conf.; Composites, Standards Testing and Design, IPC Sci. and Technol. Press, Surrey, England, 93-103.

Bentur, A., Diamond, S., and Mindess, S. (1985). "The microstructure of the steel fiber-cement interface." J. Mat. Sci., 20(10), 3610-3620.

Gare, J. M., and Timoshenko, S. P. (1984). Mechanics of materials, 2nd Ed., PWS Engineering. Boston, Mass.

Hetenyi, M. (1946). Beams on elastic foundation. Univ. of Michigan Press, Ann Arbor, Mich.

Katz, A. (1992). "Composites of fiber reinforced high-strength cementitious matrix," DSc thesis, Technion-Israel Inst. of Technol., Haifa, Israel.

Katz, A., and Bentur, A. (1994a). "Mechanical properties and pore structure of carbon fiber reinforced cementitious composite." Cement and Concrete Res., 24(2), 214-220.
Katz, A., and Bentur, A. (1994b). "Effect of matrix composition on the aging of CFRC." Int. J. Cement and Concrete Composites, 17(2), 8797.

Katz, A., and Li, V. C. (1995). "Bond properties of carbon fibers in cementitious matrix." J. Mat. in Civ. Engrg., ASCE, 7(2), 125-128.

Laws, V. (1971). "The efficiency of fibrous reinforcement of brittle matrices." J. Phys. D: Appl. Phys., 4(11), 1737-1746.

Leung, C. K. Y., and Li, V. C. (1992). "Effect of fiber inclination on crack bridging stress in brittle fiber reinforced brittle matrix composites." J. Mech. Phys. Solids, 40(6), 1333-1362.

Li, V. C. (1992). "Post crack scaling relations for fiber reinforced cementitious composites." J. Mat. in Civ. Engrg., ASCE, 4(1), 41-57.

Li, V. C., Wang, Y., and Backer, S. (1990). "Effect of inclining angle, bundling and surface treatment on synthetic fiber pull-out from a cement matrix." Composites, 21(2), 132-140.

Morton, J., and Groves, G. W. (1974). "The cracking of composites consisting of discontinuous ductile fibers in a brittle matrix-effect of fiber inclination." J. Mat. Sci., 9(9), 1436-1445.

Piggot, M. R. (1974). "Toughness in obliquely-stressed fibrous composites." J. Mech. Phys. Solids, Vol. 22, 457-468.

Soroka, I. (1979). Portland cement paste and concrete. Chemical Publishing Co., Inc., New York, N.Y.

Timoshenko, S. P. (1976). Strength of materials. II: Advanced, 3rd Ed., Van Nostrand Reinhold, New York, N.Y.

Wei, S., Mandel, J. A., and Said, S. (1986). "Study of the interface strength in steel fiber reinforced cement base composites." ACI Mat. J., 83(4), 597-605. 